\title{
MEAN APPROXIMATION ON AN INTERVAL FOR AN EXPONENT LESS THAN ONE( $\left.{ }^{1}\right)$
}

BY

T. S. MOTZKIN AND J. L. WALSH

1. Introduction. Certain properties of a least $p$ th power polynomial $p_{n}(x)$ approximating to a real continuous function $f(x)$ on a closed bounded interval $E$ are well known in the cases $p>1$ and $p=1$; these properties were established in a special case for $p=1$ by Korkine and Zolotareff, and in the general case by D. Jackson. With $p>1$ the difference $f(x)-p_{n}(x)$ must oscillate strongly at least $n+1$ times on $E$, where $n$ is the prescribed degree of the polynomial, unless that difference vanishes identically on $E$. With $p=1$ the difference must either oscillate strongly at least $n+1$ times on $E$ or vanish identically on a subset of $E$ of positive measure.

The corresponding problems for $0<p<1$ have been considered in the literature [1], [5], [6], [7], as parts of a still incomplete theory involving in terms of oscillation both necessary and sufficient conditions that a given polynomial be a polynomial of least $p$ th power approximation. The object of the present paper is to contribute to this theory, by studying in some detail approximation by a constant, namely a polynomial of degree zero. We are of the opinion that a thorough study of one nontrivial case is interesting in itself, and may well be the prelude to future deeper studies of more general cases.

Among other results, we indicate at the end of $\$ 2.6$ by a simple example that (contrary to the case $p>1$ ) with $p<1$ the difference $f(x)-p_{n}(x)$ need not oscillate strongly but may vanish only in a single point of $E$; here the zero of $f(x)-p_{n}(x)$ if unique on $E$ must be of sufficiently high order. We also indicate $(\S 3)$ that the relationship of a least $p$ th power polynomial to the function approximated may be quite complicated, even for $n=0$. However, the present writers have already established [5] necessary and sufficient conditions that a polynomial of degree $n$ should be a juxtapolynomial to $f((x)$ on $E$, and those conditions are of course necessary but are not sufficient [5] that the polynomial should be a least $p$ th power polynomial to $f(x)$ on $E$. We exhibit in the present paper some specific examples of local as well as global minima of the deviation of $p_{n}(x)$ from $f(x)$, and we are not unaware, as we shall later prove in [4] that a polynomial

Presented to the Society, July 12, 1965; received by the editors July 12, 1965.

(1) Research supported (in part) by the Office of Naval Research, U.S. Navy, and by the Office of Scientific Research, Air Research and Development Command, U.S. Air Force. 
$p_{n}(x)$ may correspond to a global maximum of the deviation even when $f(x)-p_{n}(x)$ has strong oscillations.

We call attention particularly to the contrast of the present topic with that of best approximation on a real finite point set by a polynomial $p_{n}(x)$ of arbitrary degree, with $p$ arbitrary, for which the writers have established conditions that are both necessary [2] and sufficient [3].

We emphasize, and study in $\$ 2$ in some detail, approximation by a constant to a single power of $x$ on $E$. We study the deviation, including its local analyticity, asymptotic behavior, monotonicity, and convexity. In $\S 3$ we consider application of the results just mentioned to simultaneous approximation of several powers, and in $\$ 4$ proceed to consider approximation to monotonic functions which are different from powers yet have similar geometric properties. Ensuing results on more general approximees are to be published elsewhere.

In suitable places we have included some results valid for $p \geqq 1$.

\section{Study of the deviation from a power.}

2.1. Preliminaries. We choose $f(x) \equiv x^{\alpha}, \alpha>0$, as the function to be approximated, in the interval $E: 0 \leqq x \leqq 1$. The $p$ th power deviation $(0<p<\infty)$ of the constant $c$ as approximating function from $f(x)$ is defined as

$$
\delta(c)=\int_{0}^{1}\left|x^{\alpha}-c\right|^{p} d x, \quad-\infty<c<+\infty,
$$

and is now to be studied in some detail. Clearly $\delta(c)$ is continuous for all values of $c$.

The substitution $x^{\alpha}=z, d x=\alpha^{-1} z^{\beta} d z, \beta=\alpha^{-1}-1$ yields

$$
\delta(c)=\alpha^{-1} \int_{0}^{1}|z-c|^{p} z^{\beta} d z
$$

Setting $A=(c-z)^{p} z^{\beta}, B=(z-c)^{p} z^{\beta}$ gives

$$
\begin{aligned}
& \delta(c)=\alpha^{-1} \int_{0}^{1} B d z, \quad c \leqq 0, \\
& \delta(c)=\alpha^{-1}\left(\int_{0}^{c} A d z+\int_{c}^{1} B d z\right), \quad 0 \leqq c \leqq 1, \\
& \delta(c)=\alpha^{-1} \int_{0}^{1} A d z, \quad c \geqq 1 .
\end{aligned}
$$

2.2. Piecewise analytic character. The line integral

$$
\int_{0}^{1} A d z, A=A(c, z)=\exp [p \log (c-z)+\beta \log z],
$$

for complex $c$ and $z$, depends on the path of integration and on the selection of the branches of the logarithms. 
We choose $\log z$ real, $\log c$ arbitrary, and other logarithms to satisfy $\log (1-z / c)=\log (c-z)-\log c$ and $|\operatorname{Im} \log (1-z / c)| \leqq \pi / 2$. Integration along the line segment gives the following identity on the one hand for $c$ and $\log c$ real, $c>1$, and on the other hand for all $c,|c|>1$,

$$
\begin{aligned}
\int_{0}^{1} A d z & \equiv c^{p} \int_{0}^{1}(1-z / c)^{p} z^{\beta} d z \equiv c^{p} w(c) \\
& \equiv c^{p} \int_{0}^{1} \sum_{k=0}^{\infty} C_{k}^{p}\left(-c^{-1}\right)^{k} z^{k+\beta} d z \\
& \equiv c^{p}(\beta+1)^{-1} H\left(-p, \beta+1 ; \beta+2 ; c^{-1}\right) \\
& \equiv \alpha c^{p} h(c),
\end{aligned}
$$

$h(c) \equiv H\left(1 / \alpha,-p ; 1+1 / \alpha ; c^{-1}\right) \equiv \alpha^{-1} c^{1 / \alpha} B_{1 / c}(1 / \alpha, p+1)$, where $H$ denotes a branch of the hypergeometric function and $\mathrm{B}$ a branch of the incomplete betafunction. The analytic function $H$ has 0 and 1 as its only singularities in the extended plane, and is indefinitely continuable along paths not passing through these points. The function $w(c)$ is single-valued and analytic for all $c$ with $|c|>1$, even $c=\infty$.

It is appropriate to distinguish the analytic function $w(c)$, defined for $|c|>1$, and the integral defining $w(c)$, analytic throughout the $c$-plane cut along $0 \leqq c \leqq 1$. These two functions are identical for $|c|>1$, and hence the analytic extension of $w(c)$ from $|c|>1$ exists and is equal to the integral throughout the cut plane, and each is equal there to a branch (the "principal branch") of the monogenic analytic function $\alpha h(c)$.

The analyticity for $0<c<1$ of $w(c)$ as extended analytically follows from the definition of $w(c)$ as an integral, and specifically follows from the possibility of distorting the path of integration without changing the function represented by the integral, in such a way that the path is modified only in the neighborhood of the particular real $c$ at which the analyticity is to be proved. Thus the two monogenic functions $c^{p} w(c)$ and $\alpha c^{p} h(c)$, analytic and coincident for all finite $c$ with $|c|>1$, have identical analytical extensions along every path in the cut plane commencing in $|c|>1$ and avoiding $0,1, \infty$, and are analytic and coincident even for short distances across and beyond the cut.

We now establish for suitable choice of the logarithms involved, namely $0 \leqq \arg (c-z) \leqq \pi$, the important formula

$$
\lim _{c \rightarrow c_{0}} \int_{0}^{1} A(c, z) d z=\int_{0}^{1} A\left(c_{0}, z\right) d z, \quad 0<c_{0}<1, \quad c=c_{0}+i d, \quad d \downarrow 0 .
$$

Except for the value $z=c_{0}$, we have

$$
\begin{array}{ll}
\lim _{c \rightarrow c_{0}} \log (z-c)=\log \left(z-c_{0}\right), & 0 \leqq z \leqq 1, \\
\lim _{c \rightarrow c_{0}}(z-c)^{p}=\left(z-c_{0}\right)^{p}, & 0 \leqq z \leqq 1 .
\end{array}
$$


The function $A(c, z)$ is uniformly bounded in $c$ and $z$ as $c_{0} \rightarrow c$, so the formula follows by Osgood's theorem. The integral defining $c^{p} w(c)$ is the limit of the latter function as $c$ approaches a point of the cut from above, so the integral itself is an analytic function of $c$ for every value $0<c<1$.

To study the integral in detail for $0<c<1$ we write for such values of $c$

$$
\int_{0}^{1} A d z \equiv R_{0}+R_{1}, \quad R_{0}=\int_{0}^{c}, R_{1}=\int_{c}^{1}
$$

integrals without integrand refer to the last occurring integrand. The formula for real $c$

$$
R_{0}=\alpha c^{p+1 / \alpha} \delta(1)
$$

holds, by the substitution $z=c y$, whence

$$
R_{1}=\alpha c^{p} h(c)-\alpha c^{p+1 / \alpha} \delta(1)=e^{p \pi i} \int_{c}^{1} B d z
$$

By the previous identities we have in the notation already introduced

THEOREM 1. The following formulas are valid:

$$
\begin{aligned}
\delta(c) & =(-c)^{p} h(c), \quad c<0, \\
\delta(0) & =(p \alpha+1)^{-1}, \\
\delta(c) & =(-c)^{p} h(c)+\left[1-(-1)^{p}\right] c^{p+1 / \alpha} \delta(1), \quad 0<c \leqq 1, \\
\delta(1) & =h(1)=\alpha^{-1} \Gamma(p+1) \Gamma(1 / \alpha) / \Gamma(p+1+1 / \alpha), \\
\delta(c) & =c^{p} h(c), \quad c \geqq 1, \\
h( \pm \infty) & =1,
\end{aligned}
$$

where $h$ and the powers are chosen real when possible. The function $\delta(c)$ is analytic on each of the segments $-\infty<c<0,0<c<1,1<c<+\infty$.

The only nonreal formula here is that for $\delta(c), 0<c<1, p$ not an integer. We have proved this formula with the interpretation $(-1)^{p}=e^{-p \pi i}, h(c)$ indicating the limit of the principal branch from above the cut.

3.2. Small positive $c$. For $0 \leqq c \leqq 1$ we write $c=\varepsilon^{\alpha}$, with $0 \leqq \varepsilon \leqq 1$; the relation between $c$ and $\varepsilon$ is monotonic. We set

$$
\begin{gathered}
\delta(c)=\phi(\varepsilon)=I_{1}+I_{2}, \\
I_{1}=\int_{0}^{\varepsilon}\left(\varepsilon^{\alpha}-x^{\alpha}\right)^{p} d x, \quad I_{2}=\int_{\varepsilon}^{1}\left(x^{\alpha}-\varepsilon^{\alpha}\right)^{p} d x .
\end{gathered}
$$

Substitute $x=\varepsilon z, d x=\varepsilon d z$; we obtain for $0<\varepsilon \leqq 1$ 


$$
I_{1}=\varepsilon^{\alpha p+1} I_{3}, \quad I_{3}=\int_{0}^{1}\left(1-z^{\alpha}\right)^{p} d z=I_{4}+I_{5}, \quad I_{4}=\int_{0}^{\varepsilon}, \quad I_{5}=\int_{\varepsilon}^{1}
$$

and, setting $z=y^{-1}, d z=-y^{-2} d y$,

$$
I_{2}=\varepsilon^{\alpha p+1} I_{6}, \quad I_{6}=\int_{1}^{1 / \varepsilon}\left(z^{\alpha}-1\right)^{p} d z=-\int_{1}^{\varepsilon}\left(y^{-\alpha}-1\right)^{p} y^{-2} d y=\int_{\varepsilon}^{1} .
$$

By derivation there follows

$$
\begin{aligned}
\phi^{\prime}(\varepsilon) & =(\alpha p+1) \varepsilon^{\alpha p}\left(I_{3}+I_{6}\right)-\varepsilon^{\alpha p+1}\left(\varepsilon^{-\alpha}-1\right)^{p} \varepsilon^{-2} \\
& =(a p+1) \varepsilon^{\alpha p}\left(I_{4}+I_{5}+I_{6}\right)-\varepsilon^{-1}\left(1-\varepsilon^{\alpha}\right)^{p} .
\end{aligned}
$$

Both the last term and $I_{6}$ become infinite for $\varepsilon \rightarrow 0$, partly cancelling each other as we proceed to demonstrate. First we have by integration by parts (the equations below imply that the improper integrals $I_{7}$ and $I_{8}$ converge)

$$
\begin{aligned}
& u=\left(1-z^{\alpha}\right)^{p}, \quad d v=d z, \quad d u=-\alpha p\left(1-z^{\alpha}\right)^{p-1} z^{\alpha-1} d z, \quad v=z, \\
& I_{5}=-\varepsilon\left(1-\varepsilon^{\alpha}\right)^{p}+\alpha p I_{7}, \quad I_{7}=\int_{\varepsilon}^{1}\left(1-y^{\alpha}\right)^{p-1} y^{\alpha} d y ; \\
& u=\left(1-y^{\alpha}\right)^{p}, \quad d v=y^{-\alpha p-2} d y, \quad v=(-\alpha p-1)^{-1} y^{-\alpha p-1}, \\
& I_{6}=(\alpha p+1)^{-1}\left(1-\varepsilon^{\alpha}\right)^{p} \varepsilon^{-\alpha p-1}-\alpha p(\alpha p+1)^{-1} I_{8}, \\
& I_{8}=\int_{8}^{1}\left(1-y^{\alpha}\right)^{p-1} y^{\gamma-1} d y, \quad \gamma=\alpha(1-p)-1 .
\end{aligned}
$$

Setting $I_{9}=I_{5}+I_{7}=\int_{\varepsilon}^{1}\left(1-y^{\alpha}\right)^{p-1} d y$ we have

$$
I_{5}=-\varepsilon\left(1-\varepsilon^{\alpha}\right)^{p}+\alpha p\left(I_{9}-I_{5}\right), \quad I_{5}=(1+\alpha p)^{-1}\left(-\varepsilon\left(1-\varepsilon^{\alpha}\right)^{p}+\alpha p I_{9}\right) .
$$

Writing $I_{10}=I_{9}-I_{8}=\int_{\varepsilon}^{1}\left(1-y^{\alpha}\right)^{p-1}\left(1-y^{\gamma-1}\right) d y$ (where the integrand is continuous even for $y=1$ ) we obtain finally

$$
\begin{aligned}
I_{5}+I_{6} & =(1+\alpha p)^{-1}\left(\left(1-\varepsilon^{\alpha}\right)^{p}\left(\varepsilon^{-\alpha p-1}-\varepsilon\right)+\alpha p I_{10}\right), \\
\phi^{\prime}(\varepsilon) & =(\alpha p+1) \varepsilon^{\alpha p} I_{4}+\alpha p \varepsilon^{\alpha p} I_{10}-\left(1-\varepsilon^{\alpha}\right)^{p} \varepsilon^{\alpha p+1} .
\end{aligned}
$$

We shall now study the asymptotic behavior of $\phi^{\prime}(\varepsilon)$ as $\varepsilon$ approaches 0 , which will of course depend on $\alpha$ and $p$. If $\gamma=1$, we have $I_{10}=0$ and thus (since $I_{4} \sim \varepsilon$ )

$$
\phi^{\prime}(\varepsilon) \sim(\alpha p+1) \varepsilon^{\alpha p+1}-\varepsilon^{\alpha p+1}=\alpha p \varepsilon^{\alpha p+1}=\alpha p \varepsilon^{\alpha-1} .
$$

If $\gamma \neq 1$, then $\phi^{\prime}(\varepsilon) \sim \alpha p \varepsilon^{\alpha p} I_{10}$; let

$$
I_{10}=I_{11}+I_{12}, \quad I_{11}=\int_{\varepsilon}^{1 / 2}, \quad I_{12}=\int_{1 / 2}^{1} .
$$

Further $I_{13}=\int_{\varepsilon}^{1 / 2}\left(1-y^{j-1}\right) d y=\frac{1}{2}-\varepsilon-\left(\left(\frac{1}{2}\right)^{\gamma}-\varepsilon^{\gamma}\right) / \gamma$ if $y \neq 0$, but 
$I_{13}=\frac{1}{2}-\varepsilon-\left(\log \frac{1}{2}-\log \varepsilon\right)$ for $\gamma=0$. By l'Hospital's rule, for $\gamma \leqq 0, \lim I_{11} / I_{13}=1$. Thus we have

$$
\begin{aligned}
& \text { for } \gamma<0, \quad I_{10} \sim I_{11} \sim \varepsilon^{\gamma} / \gamma ; \\
& \text { for } \gamma=0, \quad I_{10} \sim I_{11} \sim \log \varepsilon ; \\
& \text { for } \gamma>0, \quad \lim I_{10}=\mu=\mu(\alpha, p)=\int_{0}^{1}\left(1-y^{\alpha}\right)^{p-1}\left(1-y^{\gamma-1}\right) d y \text { (finite). }
\end{aligned}
$$

Here $\mu<0,=0$, or $>0$ according as $\gamma<1,=1$, or $>1$.

By the mean value theorem, $\lim _{\varepsilon \rightarrow 0} \phi^{\prime}(\varepsilon)=\phi^{\prime}(0+)$ if the limit exists, and similarly for $\delta^{\prime}(c)$. Taking into account that $\delta^{\prime}(c)=\phi^{\prime}(\varepsilon) /\left(\alpha \varepsilon^{\alpha-1}\right)$ and $\alpha p+\gamma=\alpha-1$ we obtain

THEOREM 2. For $\varepsilon \downarrow 0$ (or $c \downarrow d 0)$ we have $(0<p<1)$

(1) if $0<\alpha<1$, i.e., $-1<\gamma<-p$ : $\phi^{\prime}(\varepsilon) \sim \alpha p \varepsilon^{\alpha-1} / \gamma, \quad \phi^{\prime}(0+)=-\infty$, $\delta^{\prime}(0+)=p / \gamma<0$

(2) if $\alpha=1$, i.e., $\gamma=-p: \phi^{\prime}(0+)=\delta^{\prime}(0+)=-1$;

(3) if $1<\alpha<1 /(1-p)$, i.e., $-p<\gamma<0$ : $\phi^{\prime}(\varepsilon) \sim \alpha p \varepsilon^{\alpha-1} / \gamma<0, \phi^{\prime}(0+)=0$, $\delta^{\prime}(0+)=p / \gamma<0$

(4) if $\alpha=1 /(1-p)$, i.e., $\gamma=0$ : $\phi^{\prime}(\varepsilon) \sim \alpha p \varepsilon^{\alpha-1} \log \varepsilon<0, \quad \phi^{\prime}(0+)=0$, $\delta^{\prime}(c) \sim(p / \alpha) \log c<0, \delta^{\prime}(0+)=-\infty$;

(5) if $1 /(1-p)<\alpha<2 /(1-p)$, i.e., $0<\gamma<1$ : $\phi^{\prime}(\varepsilon) \sim \mu \alpha p \varepsilon^{\alpha p}<0, \phi^{\prime}(0+)=0$, $\delta^{\prime}(c) \sim \mu p c^{p-1+1 / \alpha}, \delta^{\prime}(0+)=-\infty$;

(6) if $\alpha=2 /(1-p)$, i.e., $\gamma=1: \phi^{\prime}(\varepsilon) \sim \alpha p \varepsilon^{\alpha-1}>0, \phi^{\prime}(0+)=0, \delta^{\prime}(0+)=p>0$;

(7) if $2 /(1-p)<\alpha<\infty$, i.e., $1<\gamma<\infty$ : $\phi^{\prime}(\varepsilon) \sim \mu \alpha p \varepsilon^{\alpha p}>0, \quad \phi^{\prime}(0+)=0$, $\delta^{\prime}(c) \sim \mu p c^{p-1+1 \alpha}, \delta^{\prime}(0+)=+\infty$.

The asymptotic values of $\phi(\varepsilon)$ and $\delta(c)$ for small positive $\varepsilon$ and $c$ follow immediately by integration and possible use of l'Hospital's rule from those for the derivatives given in Theorem 2 and from $\phi(0)=\delta(0)=1 /(1+p \alpha)$.

REMARK. For $p \geqq 1$ we have $\gamma \leqq-1$, and only cases (1) to (3) subsist. The defining relations are (1) $0<\alpha<1$; (2) $\alpha=1$; (3) $1<\alpha<\infty$, and the results are the same as in Theorem 2.

2.4. Small negative $c$. For $c \leqq 0$ we write $-c=(-\varepsilon)^{\alpha}$, with $\varepsilon \leqq 0$; the relation between $c$ and $\varepsilon$ is monotonic. We set as in 2.3

$$
\begin{gathered}
\delta(c)=\phi(\varepsilon)=\int_{0}^{1}\left(x^{\alpha}+(-\varepsilon)^{\alpha}\right)^{p} d x=J_{1}+J_{2}, \\
J_{1}=\int_{0}^{-\varepsilon}, \quad J_{2}=\int_{-\varepsilon}^{1} .
\end{gathered}
$$


Substitute $x=-\varepsilon z, d x=-\varepsilon d z$; we obtain for $\varepsilon<0$

$$
\begin{gathered}
J_{1}=(-\varepsilon)^{\alpha p+1} J_{3}, \quad J_{3}=\int_{0}^{1}\left(1+z^{\alpha}\right)^{p} d z=J_{4}+J_{5}, \\
J_{4}=\int_{0}^{-\varepsilon}, \quad J_{5}=\int_{-\varepsilon}^{1},
\end{gathered}
$$

and with $z=y^{-1}, d z=-y^{-2} d y$,

$$
J_{2}=(-\varepsilon)^{\alpha p+1} J_{6}, \quad J_{6}=\int_{1}^{-1 / \varepsilon}\left(1+z^{\alpha}\right)^{p} d z=-\int_{1}^{-\varepsilon}\left(1+y^{-\alpha}\right)^{p} y^{-2} d y=\int_{-\varepsilon}^{1} \text {. }
$$

By derivation there follows

$$
\begin{aligned}
\phi^{\prime}(\varepsilon) & =-(\alpha p+1)(-\varepsilon)^{\alpha p}\left(J_{3}+J_{6}\right)+(-\varepsilon)^{\alpha p+1}\left(1+(-\varepsilon)^{-\alpha}\right)^{p} \varepsilon^{-2} \\
& =-(\alpha p+1)(-\varepsilon)^{\alpha p}\left(J_{4}+J_{5}+J_{6}\right)+(-\varepsilon)^{-1}\left((-\varepsilon)^{\alpha}+1\right)^{p}
\end{aligned}
$$

Here we integrate by parts:

$$
\begin{aligned}
u & =\left(1+z^{\alpha}\right)^{p}, \quad d v=d z, \quad d u=\alpha p\left(1+z^{\alpha}\right)^{p-1} z^{\alpha-1} d z, \quad v=z, \\
J_{5} & =2^{p}+\varepsilon\left(1+(-\varepsilon)^{\alpha}\right)^{p}-\alpha p J_{7}, \quad J_{7}=\int_{-\varepsilon}^{1}\left(1+z^{\alpha}\right)^{p-1} z^{\alpha} d z \\
u & =\left(1+y^{\alpha}\right)^{p}, \quad d v=y^{-\alpha p-2} d y, \quad v=(-\alpha p-1)^{-1} y^{-\alpha p-1}, \\
J_{6} & =(-\alpha p-1)^{-1}\left(2^{p}-\left(1+(-\varepsilon)^{\alpha}\right)^{p}(-\varepsilon)^{-\alpha p-1}\right)-(-\alpha p-1)^{-1} \alpha p J_{8}, \\
J_{8} & =\int_{-\varepsilon}^{1}\left(1+y^{\alpha}\right)^{p-1} y^{\gamma-1} d y .
\end{aligned}
$$

We set $J_{9}=J_{5}-J_{7}=\int_{-\varepsilon}^{1}\left(1+z^{\alpha}\right)^{p-1} d z$, and obtain

$$
\begin{aligned}
& J_{5}=2^{p}+\varepsilon\left(1+(-\varepsilon)^{\alpha}\right)^{p}-\alpha p\left(J_{5}-J_{9}\right), \\
& J_{5}=(\alpha p+1)^{-1}\left[2 p+\varepsilon\left(1+(-\varepsilon)^{\alpha}\right)^{p}\right]+\alpha p(\alpha p+1)^{-1} J_{9} .
\end{aligned}
$$

We set further $J_{10}=J_{8}+J_{9}=\int_{-\varepsilon}^{1}\left(1+y^{\alpha}\right)^{p-1}\left(1+y^{\gamma-1}\right) d y$, and obtain

$$
\begin{aligned}
J_{5}+J_{6} & =(1+\alpha p)^{-1}\left(1+(-\varepsilon)^{\alpha}\right)^{p}\left(\varepsilon+(-\varepsilon)^{-\alpha p-1}\right)+\alpha p(1+\alpha p)^{-1} J_{10}, \\
\phi^{\prime}(\varepsilon) & =-(1+\alpha p)(-\varepsilon)^{\alpha p} J_{4}-\alpha p(-\varepsilon)^{\alpha p} J_{10}+\left(1+(-\varepsilon)^{\alpha}\right)^{p}(-\varepsilon)^{\alpha p+1} .
\end{aligned}
$$

We proceed to study the asymptotic behavior of $\phi^{\prime}(\varepsilon)$ as $\varepsilon \rightarrow 0, \varepsilon<0$. We clearly have $J_{4} \sim-\varepsilon$, and for $\gamma>0$ the integral $J_{10}$ approaches a finite positive limit $v=v(\alpha, p)$, so we have $\phi^{\prime}(\varepsilon) \sim-\alpha p(-\varepsilon)^{\alpha p} v$ when $\gamma>0$. When $\gamma \leqq 0$, by l'Hospital's rule, $\lim J_{10} / J_{11}=1$, where $J_{11}=\int_{-\varepsilon}^{1}\left(1+y^{\gamma-1}\right) d y$. Since $J_{11}=1+\varepsilon+\left(1-(-\varepsilon)^{\gamma}\right) / \gamma$ for $\gamma \neq 0$, but $J_{11}=1+\varepsilon-\log (-\varepsilon)$ for $\gamma=0$, we obtain by the same considerations as those preceding Theorem 2 (here $\delta^{\prime}(c)=\phi^{\prime}(\varepsilon) /\left(\alpha(-\varepsilon)^{\alpha-1}\right)$ 
THEOREM 3. For $\varepsilon \uparrow 0$ (or $c \uparrow 0$ ) we have, for (1)-(7) defined in Theorem 2, and as in the Remark thereto,

(1): $\phi^{\prime}(\varepsilon) \sim \alpha p(-\varepsilon)^{a-1} / \gamma, \phi^{\prime}(0-)=-\infty, \delta^{\prime}(0-)=p / \gamma<0$;

(2): $\phi^{\prime}(0-)=\delta^{\prime}(0-)=-1$;

(3): $\phi^{\prime}(\varepsilon) \sim \alpha p(-\varepsilon)^{\alpha-1} / \gamma, \phi^{\prime}(0-)=0, \delta^{\prime}(0-)=p / \gamma<0$;

(4): $\phi^{\prime}(\varepsilon) \sim \alpha p(-\varepsilon)^{\alpha-1} \log (-\varepsilon)<0, \phi^{\prime}(0-)=0, \delta^{\prime}(c) \sim(p / \alpha) \log (-c)<0$, $\delta^{\prime}(0-)=-\infty$;

(5)-(7): $\phi^{\prime}(\varepsilon) \sim-v \alpha p(-\varepsilon)^{\alpha p}<0, \quad \phi^{\prime}(0-)=0, \quad \delta^{\prime}(c) \sim-v p(-c)^{p-1+1 / \alpha}$, $\delta^{\prime}(0-)=-\infty$.

The asymptotic values of $\phi(\varepsilon)$ and $\delta(c)$ follow as after Theorem 2 .

The limit $\delta^{\prime}(c)$ as $c(<0) \rightarrow 0$ can also be studied directly. We have $(c<0)$

$$
\delta(c)=\frac{1}{\alpha} \int_{0}^{1}(z-c)^{p} z^{-1+1 / \alpha} d z, \quad \delta^{\prime}(c)=-\frac{p}{\alpha} \int_{0}^{1}(z-c)^{p-1} z^{-1+1 / \alpha} d z .
$$

As $c$ approaches zero monotonically, $(z-c)^{p-1} \rightarrow z^{p-1}$, so

$$
\delta^{\prime}(0-)=-\frac{p}{\alpha} \int_{0}^{1} z^{p-2+1 / \alpha} d z=\frac{p}{\gamma}
$$

provided $\gamma<0$, that is, provided the last integral converges. In the contrary case $(p+1 / \alpha \leqq 1)$ we have $\delta^{\prime}(c) \rightarrow-\infty$ and by the law of the mean $\delta^{\prime}(0-)=-\infty$.

2.5. Behavior near $c=1$. We prove

THEOREM 4. We have for $p<1$

$$
\delta^{\prime}(1)=p \int_{0}^{1}\left(1-x^{\alpha}\right)^{p-1} d x
$$

which is finite and positive,

$$
\delta^{\prime \prime}(1-)=+\infty, \quad \delta^{\prime \prime}(1+)=-\infty .
$$

The values for $\delta^{\prime}(1+)$ and $\delta^{\prime \prime}(1+)$ follow by use of the mean value theorem from the values $(c>1)$

$$
\delta^{\prime}(c)=p \int_{0}^{1}\left(c-x^{\alpha}\right)^{p-1} d x, \quad \delta^{\prime \prime}(c)=p(p-1) \int_{0}^{1}\left(c-x^{\alpha}\right)^{p-2} d x .
$$

In order to study $\delta(c), 0<c<1$, we set

$$
\phi^{\prime}(\varepsilon) \equiv \varepsilon^{\alpha p} \psi(\varepsilon), \quad \psi(\varepsilon) \equiv(\alpha p+1) I_{4}+\alpha p I_{10}-\left(1-\varepsilon^{\alpha}\right)^{p} \varepsilon
$$

differentiation yields $\psi^{\prime}(\varepsilon)=\alpha p \varepsilon^{\gamma-1}\left(1-\varepsilon^{\alpha}\right)^{p-1}$. Hence we have $\phi^{\prime}(1-)=(\alpha p+1) I_{3}$ since $I_{10} \rightarrow 0$. Integration of $I_{3}$ by parts with $u=\left(1-x^{\alpha}\right)^{p}, d v=d x$, shows

$$
I_{3}=\frac{\alpha p}{\alpha p+1} \int_{0}^{1}\left(1-x^{\alpha}\right)^{p-1} d x,
$$

whence $\delta^{\prime}(1-)=\phi^{\prime}(1-) / \alpha$ has the asserted value. 
By the definition of $\psi(\varepsilon)$ there follows

$$
\phi^{\prime \prime}(\varepsilon) \equiv \varepsilon^{\alpha p} \psi^{\prime}(\varepsilon)+\alpha p \varepsilon^{\alpha p-1} \psi(\varepsilon) .
$$

Since $\psi(1-)=\phi^{\prime}(1-)$ is finite and $\psi^{\prime}(1-)=+\infty$, we have $\phi^{\prime \prime}(1-)=+\infty$, whence also $\delta^{\prime \prime}(1-)=+\infty$.

2.6. Piecewise monotonicity of $\delta(c)$. Clearly $\delta(c)$ decreases for $c<0$ and increases for $c>1$. The minimum of the continuous function $\delta(c)$ thus exists and is attained in the interval $0 \leqq c \leqq 1$.

By another use of the mean value theorem, to evaluate $\phi(\varepsilon)-\phi(0)$ or $\delta(c)-\delta(0)$, there follows for $p<1$ from Theorem 2 that each of the functions $\phi(\varepsilon), 0 \leqq \varepsilon \leqq 1$, and $\delta(c), 0 \leqq c \leqq 1$, has a local maximum or minimum at $\varepsilon=0$ and $c=0$ according as $\alpha<2 /(1-p), \gamma<1$, or $\alpha \geqq 2 /(1-p), \gamma \geqq 1$.

For $p \geqq 1$ these functions have a local maximum at $\varepsilon=0$ and $c=0$.

We shall now prove

THEOREM 5. There exists $c_{p}=c_{p}(\alpha), 0 \leqq c_{p}<1$, such that $\delta(c)$ strictly decreases for $-\infty<c<c_{p}$ and strictly increases for $c_{p}<c<\infty$. Thus $\delta(c)$ has a single global minimum; this occurs at $c=0$ if and only if $p<1, \alpha \geqq 2 /(1-p)$ (i.e., $\gamma \geqq 1$ ).

As indicated before we need merely consider $0 \leqq c \leqq 1$. Suppose $\gamma \geqq 1$; then $I_{10}>0$ for $0<\varepsilon<1$. The inequality $I_{4}>\varepsilon\left(1-\varepsilon^{\alpha}\right)^{p}$ is obvious, so we have $\phi^{\prime}(\varepsilon)>\alpha p \varepsilon^{\alpha p}\left(I_{4}+I_{10}\right)>0$, which implies the conclusion for this case, with $c_{p}=0$.

For arbitrary $\alpha(>0)$ or $\gamma(>-1), \psi^{\prime}(\varepsilon)$ is positive, so $\psi(\varepsilon)$ is strictly increasing and therefore vanishes for at most one value of $\varepsilon, 0<\varepsilon<1$. Thus $\phi^{\prime}(\varepsilon)$ vanishes for at most one such value of $\varepsilon$, and is locally increasing there. Consequently $\phi(\varepsilon)$ has no local maximum in $0<\varepsilon<1$; therefore $\delta(c)$ has no local maximum in $-\infty<c<\infty$, and the existence of $c_{p}$ as in Theorem 5 is assured; Theorem 3 implies $c_{p}<1$. The inequality $c_{p}>0$ for $\gamma<1$ follows from Theorem 2 and the Remark thereto.

It is a consequence of Theorem 5 that under suitable conditions $\delta(c)$ has a global minimum for $c=0$. Thus for arbitrary $p(<1)$ there exists a function $f(x)$ analytic on $0 \leqq x \leqq 1$, and a polynomial $p_{0}(x) \equiv 0$ of degree zero, such that $p_{0}(x)$ is the polynomial of degree zero of best approximation to $f(x)$ on the interval in the sense of least $p$ th powers, yet $f(x)-p_{0}(x)$ has neither one strong sign change on the interval nor coincides with $f(x)$ on a subset of positive measure. This is in great contrast to the classical results for $p>1$ and $p=1$.

2.7. Convexity and concavity. For $p>1, \delta(c)$ is strictly convex, $-\infty<c<\infty$, being the integral (with respect to $x$ ) of the strictly convex function $\left|c-x^{\alpha}\right|^{p}$ of $c$. For $p=1$ we see similarly that $\delta(c)$ is (not necessarily strictly) convex; direct evaluation shows that strict convexity holds precisely in $0<c<1$. 
Near $c= \pm \infty, \delta(c)$ behaves for all $p>0$ (e.g., by Theorem 1) like $|c|^{p}$. In the intervals $c>1$ and $c<0$ we have $\delta^{\prime \prime}(c)=p(p-1) \int_{0}^{1}\left|c-x^{\alpha}\right|^{p-2} d x$; for $p<1$, $\delta(c)$ is concave there since $\delta^{\prime \prime}(c)<0$. Near $1-, \delta(c)$ is always convex (Theorem 4).

From $\delta^{\prime}(c)=\alpha^{-1} \varepsilon^{-\gamma} \psi(\varepsilon)$ we see that $\delta(c)$ is convex in $c_{p}<c<1$ if $\gamma \leqq 0$ and in $0<c<c_{p}$ if $\gamma \geqq 0$. For $\alpha=1 \quad(\gamma=-p), 0<c<1, \delta(c)=\left(c^{p+1}+(1-c)^{p+1}\right) /(p+1)$ is convex; as $\delta^{\prime \prime}(c)=p\left(c^{p-1}+(1-c)^{p-1}\right)$ is bounded away from 0 we see that $\delta(c), 0<c<1$, is convex for all $\alpha$ sufficiently near 1 . For $p<1, \alpha>2 /(1-p)$, i.e., $\gamma>1$, Theorem 2 implies that $\delta(c)$ cannot be convex for all small $c>0$.

The function $\phi(\varepsilon)$ is convex in $\varepsilon_{p}\left(=c_{p}^{1 / \alpha}\right)<\varepsilon<1$, since $\phi^{\prime}(\varepsilon)>0$ implies $(\S 2.4) \phi^{\prime \prime}(\varepsilon)>0$. For $1<\alpha<2 /(1-p)$ we have $\phi^{\prime}(0+)=0$ yet $\phi^{\prime}(\varepsilon)<0$ for small positive $\varepsilon$ and thus $\phi(\varepsilon)$ is not convex in the neighborhood of 0 .

3. Simultaneous approximation of powers. Let $\delta(c, a, b)=\int_{0}^{b}\left|c-a x^{\alpha}\right|^{p} d x$ denote the $p$ th power deviation of the constant $c$ from the function $a x^{\alpha}(a \neq 0$, $\alpha>0), 0 \leqq x \leqq b(b>0)$; then $\delta(c, 1,1)$ is our former $\delta(c)$. By use of the substitution $x=b y$ we see that $\delta(c, a, b)=|a|^{p} b^{\alpha p+1} \delta\left(c, a^{-1}, b^{-\alpha}\right)$.

Now let the function $f(x)$ be defined in the interiors (assumed mutually disjoint) of finitely many intervals $x=x_{k}+\theta_{k} b_{k}, 0 \leqq \theta_{k} \leqq 1, b_{k} \neq 0$, as $a_{k}\left|x-x_{k}\right|^{\alpha_{k}}$, $a_{k} \neq 0, \alpha_{k}>0$, respectively. Then the deviation of the constant $c$ from $f$ is $\delta(c, f)$ $=\Sigma_{k}\left|a_{k}\right|^{p}\left|b_{k}\right|^{\alpha_{k} p+1} \delta\left(c a_{k}^{-1}\left|b_{k}\right|^{-\alpha_{k}}\right)$.

We arrange the $\alpha_{k}$ so that all $\alpha_{k}=\beta /(1-p)$ with $a_{k}>0$ come last, and that among the other $\alpha_{k}$ the largest ones, $\alpha_{1}=\cdots=\alpha_{j}$, come first. By Theorems 1, 2, and 3 we obtain

THEOREM 6. The function $\delta(c, f)$ of $c$ is piecewise analytic. If $p<1$, $\alpha=\alpha_{1}>1 /(1-p)\left(\right.$ but not $\left.\alpha=2 /(1-p), a_{1}>0\right)$ and if

$$
\begin{gathered}
\lambda=\mu(\alpha, p) a+v(\alpha, p) a^{\prime} \neq 0, \\
a=\sum_{k \leqq j, a_{k}>0} a_{k}^{-1 / \alpha}, \quad a^{\prime}=\sum_{k \leqq j, a_{k}<0}\left(-a_{k}\right)^{-1 / \alpha},
\end{gathered}
$$

then $\delta(c, f)-\delta(0, f) \sim \lambda p c^{p+1 / \alpha} /(p+1 / \alpha)$ for small positive $c ;$ if $\alpha=1 /(1-p)$ and if

$$
\lambda=a^{\prime}-a \neq 0
$$

then $\delta(c, f)-\delta(0, f) \sim-\lambda p(1-p) c \log c ; \quad$ if $\quad \alpha=2 /(1-p), \quad a_{1}>0, \quad$ or $\alpha<1 /(1-p)$ or $p \geqq 1$, and if

$$
\lambda=\sum_{k} \gamma_{k}^{-1}\left|a_{k}\right|^{p-1}\left|b_{k}\right|^{-\gamma_{k}} \operatorname{sg} a_{k} \neq 0, \quad \gamma_{k}=\alpha_{k}(1-p)-1,
$$

then $\delta(c, f)-\delta(0, f) \sim \lambda p c$.

Theorem 5 implies 
THEOREM 7. If $p<1$ and each $\alpha_{k} \geqq 2 /(1-p)$, then $\delta(c, f)$ and $\delta(-c, f)$ increase with $c$ for $0<c<\infty$, and thus $c=0$ is the best constant global approximator, in the sense of least pth powers.

From Theorem 6 follows

THEOREM 8. At $c=0$ there is a local minimum of $\delta(c, f), c \geqq 0$, if $\lambda>0$ but not if $\lambda<0$. A two-sided local minimum of $\delta(c, f)$ for $p \geqq 1$ requires $\lambda=0$; for $p<1$ and each $\alpha_{k} \neq 2 /(1-p)$, with $\lambda \neq 0, a$ two-sided local minimum occurs if and only if either $\alpha_{1}>2 /(1-p)$, or $2 /(1-p)>\alpha_{1}>1(1-p)$ and $\min \left(a / a^{\prime}, a^{\prime} \mid a\right)>-\mu\left(\alpha_{1}, p\right) / v\left(\alpha_{1}, p\right)$.

That $|\mu / v|<1$ follows from the following

LEMMA. Let $p<1$. Then the function $\mu(\alpha)=\mu(\alpha, p)$ increases strictly with $\alpha$ for $1 /(1-p)<\alpha<\infty$ from $-\infty$ to 1 ; we have

$$
\begin{gathered}
\mu(\alpha)=\Gamma(p) \Gamma(1+1 / \alpha)(1-\sin \pi / \alpha / \sin (p+1 / \alpha) \pi) / \Gamma(p+1 / \alpha), \\
\mu(2 /(1-p))=0 .
\end{gathered}
$$

The function $v(\alpha)=v(\alpha, p)$ decreases strictly for $1 /(1-p)<\alpha<\infty$ from $+\infty$ to 1 , and $\mu(\alpha)+v(\alpha)$ increases strictly for $1 /(2-p)<\alpha<\infty$ from $-\infty$ to 2 , passing through 0 when $\alpha=1$.

Here $\mu(\alpha)+v(\alpha)$ means the value at 0 of $I_{10}+J_{10}$ even when $I_{10}$ and $J_{10}$ separately diverge there.

To prove the lemma note that, for $\eta= \pm 1$,

$$
d\left(1+\eta y^{\alpha}\right)^{p-1}\left(1+\eta y^{\gamma-1}\right) / d \alpha=\eta(1-p)\left(1+\eta y^{\alpha}\right)^{p-2}\left(y^{\gamma-1}-y^{\alpha}\right) \log y
$$

implies the monotonicity properties stated. Setting $y^{\alpha}=z$ we have $I_{10}+J_{10}=$ $K+\alpha^{-1} \int_{c}^{1} z^{-p-1 / \alpha}\left((1+z)^{p-1}-(1-z)^{p-1}\right) d z=2 \alpha^{-1} \int_{c}^{1}\left((p-1) z^{1-p-1 / \alpha}\right.$ $\left.+z^{3-p-1 / \alpha} m(z)\right) d z$ where $K$ and $m(z)$ are bounded, whence for $\varepsilon \downarrow 0, I_{10}+J_{10}-K \sim 2(1-p)(2 \alpha-\alpha p-1)^{-1} \varepsilon^{2 \alpha-\alpha p-1}$ unless $\alpha=1 /(2-p)$, in which case $I_{10}+J_{10} \sim 2(1-p) \log \varepsilon$. For $\alpha \rightarrow+\infty$ and fixed small positive $\theta$ we have $\int_{0}^{1-\theta}\left(1+\eta y^{\alpha}\right)^{p-1}\left(1+\eta y^{\gamma-1}\right) d y \rightarrow 1-\theta$ while, in $1-\theta<y<1$, the integrand is $<2$ for $\eta=1$ and $\alpha>2 /(1-p)$, $<1$ for $\eta=-1$; the latter follows from $1-y^{\gamma-1}<1-y^{\alpha}<\left(1-y^{\alpha}\right)^{1-p}$. For $\alpha=1$ we have $\int_{\varepsilon}^{1}(1+\eta y)^{p-1}\left(1+\eta y^{-p-1}\right) d y=\eta(1+\eta y)^{p}\left(1-y^{-p}\right) /\left.p\right|_{\varepsilon} ^{1}$. By the law of the mean for $x^{p}, 0<r_{1}<x<r_{2}$, there follows $\left(r_{2}-r_{1}\right) p r_{2}^{p-1}<r_{2}^{p}-r_{1}^{p}<\left(r_{2}-r_{1}\right) p r_{1}^{p-1}$. Hence $2 \varepsilon p(1+\varepsilon)^{p-1}<(1+\varepsilon)^{p}-(1-\varepsilon)^{p}<2 \varepsilon p(1-\varepsilon)^{p-1}, 2\left(\varepsilon^{1-p}-\varepsilon\right)(1+\varepsilon)^{p-1}$ $<I_{10}+J_{10}<2\left(\varepsilon^{1-p}-\varepsilon\right)(1-\varepsilon)^{p-1}, I_{10}+J_{10} \sim 2 \varepsilon^{1-p}$. The expression for $\mu(\alpha)$ is obtained by substituting $y^{\alpha}=z$ and using well-known formulas for the $\Gamma$-function.

Special evaluation of $\delta(c, f)-\delta(0, f)$ is needed when $\lambda=0$. For the case of two intervals with $a_{1}=-a_{2}=b_{1}=b_{2}=1, \lambda=0$ implies $\alpha_{1}=\alpha_{2}$. We prove 
THEOREM 9. For $f(x)=|x|^{\alpha} \operatorname{sg} x,-1 \leqq x \leqq 1$, we have, for $p<1$ and $c \rightarrow 0$, $\delta(c, f)-\delta(0, f) \sim p(1-p)(2 \alpha-\alpha p-1)^{-1} c^{2}, \quad p(1-p) c^{2} \log |c|, \quad p(\mu+v)$ $\cdot|c|^{p+1 / \alpha} /(p+1 / \alpha), p c^{2}, p(\mu+v)|c|^{p+1 / \alpha} /(p+1 / \alpha)$ respectively for $\alpha<1 /(2-p)$, $\alpha=1 /(2-p), 1 /(2-p)<\alpha<1, \alpha=1,1<\alpha$. Thus at $c=0$ there is a local strict maximum or minimum according as $\alpha<1$ or $\alpha \geqq 1$.

Indeed, from the formulas in the proof of the lemma we see that for $c \rightarrow 0$ the term involving $I_{10}+J_{10}$ in $\delta(c, f)-\delta(0, f)$ dominates the others, even when $\mu+\nu=0$, and we obtain the asymptotic expression of this term as stated in the theorem.

With $p \geqq 1$, the even function $\delta(c, f)$ for $f$ as in Theorem 9 has a strict minimum for $c=0$. Here $(p<1) \delta(c, f)$ exhibits a local strict maximum for $c=0$, which is a rarity in the theory of appproximation on an interval. This phenomenon will be further studied in [4].

\section{Deviation from a monotonic function.}

4.1. Piecewise analyticity. We proceed to study the deviaton $\delta(c)$ on the interval $0 \leqq x \leqq 1$ for approximation to an arbitrary continuous monotonic function $\alpha(x)$ by a constant $c$, in a manner analogous to that of $\S 2$. We suppose $\alpha(0)=0, \alpha(1)=1$, and denote the inverse of $z=\alpha(x)$ by $x=\beta(z), 0 \leqq z \leqq 1$. The deviation

$$
\delta(c)=\int_{0}^{1}|c-\alpha(x)|^{p} d x=\int_{0}^{1}|c-z|^{p} d \beta(z)
$$

is clearly continuous for all values of $c$. With $A \equiv(c-z)^{p} d \beta(z), B \equiv(z-c)^{p} d \beta(z)$, we have

$$
\delta(c)=\int_{0}^{1} B, \quad \int_{0}^{c} A+\int_{c}^{1} B, \int_{0}^{1} A,
$$

accordings as $c \leqq 0,0 \leqq c \leqq 1, c \geqq 1$. For complex $c$ and $z$ we define $A \equiv \exp [p \log (c-z)] d \beta(z)$. Then $\int_{0}^{1} A$ depends on the path of integration and on the branch of the $\operatorname{logarithm}$. We choose $\log c$ arbitrary and other logarithms to satisfy $\log (1-z / c)=\log (c-z)-\log c$ and $|\arg (1-z / c)|<\pi$. Integration along the line segment gives for all $c$ outside the segment $0 \leqq c \leqq 1$

$$
\int_{0}^{1} A \equiv c^{p} \int_{0}^{1}\left(1-\frac{z}{c}\right)^{p} d \beta(z) \equiv c^{p} w(c) .
$$

The function $w(c)$ is single-valued and analytic in the cut plane even at $c=\infty$, as follows by differentiation under the integral sign; we have $w(\infty)=1$.

The properties of $w(c)$ obviously depend heavily on those of $\beta(z)$. (i) Let $\beta^{\prime}(z)$ exist and be continuous, $0 \leqq z \leqq 1$. There exists (Weierstrass) a uniform development of $\beta^{\prime}(z)$ on $0 \leqq z \leqq 1$ in polynomials in $z$ : 


$$
\begin{aligned}
\beta^{\prime}(z) & \equiv \sum_{n=0}^{\infty} p_{n}(z), \quad p_{n}(z) \equiv \sum_{k=0}^{n} a_{n k} z^{k}, \\
\int_{0}^{1} A & =\int_{0}^{1}(c-a)^{p} \sum_{0}^{\infty} \sum_{k=0}^{n} a_{n k} z^{k} d z \\
& \equiv c^{p} \sum_{n=0}^{\infty} \sum_{k=0}^{n} a_{n k} H\left(-p, k+1 ; k+2 ; c^{-1}\right) \\
& \equiv c^{p} \sum_{n=0}^{\infty} \sum_{k=0}^{n}(k+1) a_{n k} c^{k+1} \mathbf{B}_{1 / c}(k+1, p+1),
\end{aligned}
$$

an expansion valid for all finite $c$. (ii) If $\beta^{\prime}(z)$ is analytic at a point $c_{0}$ of the segment $0 \leqq z \leqq 1$, the path of integration can be slightly distorted in the neighborhood of $c_{0}$, as in 2.2 , so the monogenic function $w(c)$ can be extended analytically from either bank of the segment across the segment at $c_{0}$ and slightly beyond. The function $w(c)$ as extended need not be single-valued throughout a neighborhood of $c_{0}$. (iii) Even if $\beta^{\prime}(z)$ is not analytic on the segment, it may be possible to extend $w(c)$ across the segment. Indeed, if $p$ is an integer, then $c^{p} w(c)$ is a polynomial in $c$. (iv) On the other hand, a singularity of $\beta^{\prime}(z)$ at a point $c_{0}$ of the interval may block the analytic extension of $w(c)$ across the cut at $c_{0}$. As an example we choose $\beta(z) \equiv \beta_{1}(z)+\beta_{2}(z)$, where $\beta_{1}(z) \equiv z / 2, \quad 0 \leqq z \leqq 1, \beta_{2}(z) \equiv 0$ for $0 \leqq z \leqq c_{0} \leqq 2 / 3, \beta_{2}(z)=(3 z-2) / 2$ for $2 / 3 \leqq z \leqq 1$. It follows that $c^{p} w(c)$ is the sum of two integrals relating respectively to $\beta_{1}$ and $\beta_{2}$, of which the first has no singularity at $c_{0}$, while the second has a singularity for nonintegral $p$.

Precisely as in $\$ 2.2$ there follows

$$
\lim _{c \rightarrow c_{0}} \int_{0}^{1} A(c, z)=\int_{0}^{1} A\left(c_{0}, z\right), \quad 0<c_{0}<1, \quad c=c_{0}+i d, \quad d \downarrow 0 ;
$$

here we set $\int_{0}^{1} A=c_{0}^{p} w\left(c_{0}\right)$. If $\beta(z)$ is analytic for $0<z<1$, the monogenic function $w(c)$ is analytic for $0<c<1$, so the integral $\int_{0}^{1} A(c, z)$ represents the analytic function $c^{p} w(c), 0<c<1$. As before we have independently of the analyticity of $\beta(z)$

$$
\int_{0}^{1} A=R_{0}+R_{1}, \quad R_{0}=\int_{0}^{c} A, \quad R_{1}=\int_{c}^{1} A=e^{p \pi i} \int_{c}^{1} B .
$$

If $\beta(z)$ is analytic for $0<z<1$, both $R_{0}$ and $R_{1}$ are analytic functions of $c$, for their derivatives with respect to nonreal $c$ exist (and are continuous). We collect some of the previous and immediately following results in

THEOREM 10. The following formulas are valid: 


$$
\begin{aligned}
\delta(c) & =(-c)^{p} w(c), \quad c<0, \\
\delta(c) & =(-c)^{p} w(c)+\left[1-(-1)^{p}\right] R_{0}, \quad 0<c<1, \\
\delta(c) & =c^{p} w(c), \quad c \geqq 1, \\
w( \pm \infty) & =1,
\end{aligned}
$$

where the powers are chosen real when possible. If $\beta^{\prime}(z)$ is analytic for $0<z<1$, then $\delta(c)$ is analytic on each of the segments $-\infty<c<0,0<c<1,1<c<+\infty$.

We have proved the formula for $\delta(c), 0<c<1$, with the interpretation $(-1)^{p}=e^{-p \pi i}$, and where $w(c)$ indicates the limit value on the cut from above.

If $p$ is an even integer we have $\delta(c)=c^{p} w(c)$, a polynomial of degree $p$, for $-\infty<c<+\infty$. It is a striking but easily verified fact that if $p$ is an odd integer (even though $w(c)$ is a polynomial) $\delta(c)$ is analytic for $0<c<1$ if and only if $\beta(z)$ is analytic for $0<z<1$, and is a polynomial in $c$ if and only if $\beta(z)$ is a polynomial in $z$; in the latter case the degree of $\delta(c)$ exceeds that of $\beta(z)$ by $p$.

4.2. Small c. In 4.2, corresponding to 2.3 and 2.4 , we turn our attention to the asymptotic behavior of $\delta(c)$ for $c$ approaching zero, with $0<p<\infty$. We consider approximation to a function of the form $f(x) \equiv f_{x} \equiv x^{\alpha} g_{x}$ (henceforth subscripts of this kind denote functional dependence and not partial differentiation), where $f_{x}$ has a continuous positive derivative in $0<x \leqq 1, \lim _{x \rightarrow 0} g_{x}$ $=g_{0}=g_{1}=1,\left|g_{x}^{\prime}\right| \leqq G_{1}$ for some $G_{1}$, and $\alpha>0$. For $|c|<1$, we define $\varepsilon$ by the equations $|c|=f_{|\varepsilon|}, \operatorname{sg} c=\operatorname{sg} \varepsilon=s$, and set $x=|\varepsilon| z, z=1 / y, \beta=\alpha p+1$, $\gamma=\alpha-\beta$. We shall also use the abbreviations $u=u_{z}=g_{|\varepsilon|}-s z^{\alpha} g_{|\varepsilon| z}$, $v=v_{y}=g_{|\varepsilon| / y}-s y^{\alpha} g_{|\varepsilon|}=-s u_{z} y^{\alpha}$; one has $d u / d z=-s \alpha z^{\alpha-1} g_{|\varepsilon| z}-\varepsilon z^{\alpha} g_{|\varepsilon| z}^{\prime}$, $d v / d y=-s \alpha y^{\alpha-1} g_{|\varepsilon|}-|\varepsilon| y^{-2} g_{|\varepsilon| / y}^{\prime}$. We note

$$
\begin{array}{ll}
u_{z} \equiv|\varepsilon|^{-\alpha}\left(f_{|\varepsilon|}-s f_{|\varepsilon| z}\right)>0 & \text { for } 0<z<1, \\
v_{y} \equiv(y /|\varepsilon|)^{\alpha}\left(f_{|\varepsilon| / y}-s f_{|\varepsilon|}\right)>0 & \text { for }|\varepsilon|<y<1,
\end{array}
$$

$u_{1}=v_{1}>0$ or $=0$ as $\varepsilon<0$ or $>0$.

With similar but not identical notation to that of 2.3, we set

$$
\begin{aligned}
& \delta_{c}=\phi_{\varepsilon}=\int_{0}^{1}\left|c-f_{x}\right|^{p} d x=|\varepsilon|^{\beta} I_{0}, \\
& I_{0}=\int_{0}^{1 /|\varepsilon|}|u|^{p} d z=I_{4}+I_{5}+I_{6}, \\
& I_{4}=\int_{0}^{|\varepsilon|}, \quad I_{5}=\int_{|\varepsilon|}^{1}, \quad I_{6}=\int_{1}^{1 /|\varepsilon|} .
\end{aligned}
$$

Integration by parts yields 


$$
\begin{aligned}
& I_{5}=\int_{|\varepsilon|}^{1} u^{p} d z=\left.u^{p} z\right|_{|\varepsilon|} ^{1}-\int_{|\varepsilon|}^{1} z d\left(u^{p}\right)=u_{1}^{p}-|\varepsilon| u_{|\varepsilon|}^{p}+s \alpha p I_{7}, \\
& I_{7}=\int_{|\varepsilon|}^{1} u^{p-1} z^{\alpha}\left(g_{x}+\alpha^{-1} x g_{x}^{\prime}\right) d z ;
\end{aligned}
$$

the equations imply for $\varepsilon>0, p<1$, that this improper integral converges. Likewise, with $w=-\beta^{-1} y^{-\beta}$ we set

$$
\begin{aligned}
I_{6} & =\int_{1}^{1 /|\varepsilon|}(-s u)^{p} d z=\int_{|\varepsilon|}^{1} v^{p} y^{-\beta-1} d y=\left.v^{p} w\right|_{|\varepsilon|} ^{1}-\int_{|\varepsilon|}^{1} w d\left(v^{p}\right), \\
I_{6} & =\beta^{-1}\left(-v_{1}^{p}+|\varepsilon|^{-\beta} v_{|\varepsilon|}^{p}-s \alpha p g_{|\varepsilon|} I_{-2}-|\varepsilon| p I_{-4}\right), \\
I_{-2} & =\int_{|\varepsilon|}^{1} v^{p-1} y^{\gamma-1} d y, \quad I_{-4}=\int_{|\varepsilon|}^{1} v^{p-1} y^{-\beta-2} g_{x}^{\prime} d y
\end{aligned}
$$

the integral $I_{-4}$ converges for $\varepsilon>0, p<1$, provided $I_{-2}$ converges; the convergence of $I_{-2}$ follows from (4.2) by $f^{\prime}>0$.

We define further $I_{9}=I_{5}+s I_{7}=g_{|\varepsilon|} I_{-1}+\alpha^{-1} \varepsilon I_{-3}$,

$$
I_{-1}=\int_{|\varepsilon|}^{1} u^{p-1} d z, \quad I_{-3}=\int_{|\varepsilon|}^{1} u^{p-1} z^{\alpha+1} g_{x}^{\prime} d z ;
$$

here $I_{-3}$ converges for $\varepsilon>0, p<1$, because $I_{-1}$ converges, and $I_{-1}$ converges by (4.1) and $f^{\prime}>0$. Elimination of $I_{-7}$ yields

$$
I_{5}=\beta^{-1}\left(u_{1}^{p}-|\varepsilon| u_{|\varepsilon|}^{p}+\alpha p g_{|\varepsilon|} I_{-1}+p \varepsilon I_{-3}\right) \text {. }
$$

Now we obtain by differentiation

$$
\begin{aligned}
\phi_{\varepsilon}^{\prime}= & s \beta|\varepsilon|^{\beta-1}\left(I_{4}+I_{5}+I_{6}\right) \\
& +s|\varepsilon|^{\beta}\left[-v_{|\varepsilon|}^{p}|\varepsilon|^{-\beta-1}+p\left(g_{|\varepsilon|}^{\prime} I_{-5}-s I_{-3}-s I_{-7}+I_{-4}-s g_{|\varepsilon|}^{\prime} I_{-2}\right)\right],
\end{aligned}
$$

where

$$
I_{-5}=\int_{0}^{1} u^{p-1} d z, \quad I_{-7}=\int_{0}^{|\varepsilon|} u^{p-1} z^{\alpha+1} g_{x}^{\prime} d z .
$$

From equations (4.3) and (4.4) we have

$$
\begin{gathered}
\phi_{\varepsilon}^{\prime}=|\varepsilon|^{\beta-1}(\beta-1) g_{|\varepsilon|} I_{-6}+s \varepsilon^{\beta-1} \beta I_{4} \\
+\left|\varepsilon^{\beta}\right|\left[p\left(g_{|\varepsilon|}^{\prime} I_{-5}-s g_{|\varepsilon|}^{\prime} I_{-2}-s I_{-7}\right)-s u_{|\varepsilon|}^{p}\right], \\
I_{-6}=s I_{-1}-I_{-2} .
\end{gathered}
$$

If for $\varepsilon \rightarrow 0$ the quotient $1 / I_{-6}$ remains bounded, then the first term in the second 
member of (4.5) dominates the others. Indeed, because of the boundedness of $\left|g_{x}^{\prime}\right|$, it is easy to see that $u /\left(1-s z^{\alpha}\right)$ and $v /\left(1-s y^{\alpha}\right)$ are uniformly bounded and uniformly bounded from zero in $\varepsilon$ and $z$ (respectively $y$ ) for sufficiently small $\varepsilon$. Hence $I_{4}=O(\varepsilon), I_{-7}=O\left(|\varepsilon|^{\alpha+2}\right), I_{-2}=O\left(|\varepsilon|^{\min 0, \gamma}\right)$, while $I_{-1}$ and $u_{|\varepsilon|}{ }^{p}$ are $O(1)$.

To explore the behavior of $g_{|\varepsilon|} I_{-6}$ we write from (4.6)

$$
\begin{aligned}
& I_{-6}=s I_{10}+(p-1)\left(s I_{-9}-I_{-8}\right), \\
& I_{10}=\int_{|\varepsilon|}^{1}\left(1-s y^{\alpha}\right)^{p-1}\left(1-s y^{\gamma-1}\right) d y, \\
& I_{-9}=\int_{|\varepsilon|}^{1} g^{*}\left(1-s z^{\alpha}+\theta_{z} g^{*}\right)^{p-2} d z, \quad 0<\theta_{z}<1, \\
& g^{*}=g_{|\varepsilon|}-1-s z^{\alpha}\left(g_{|\varepsilon| z}-1\right), \\
& I_{-8}=\int_{|\varepsilon|}^{1} g^{* *}\left(1-s y^{\alpha}+\eta_{y} g^{* *}\right)^{p-2} y^{\gamma-1} d y, \\
& g^{* *}=g_{|\varepsilon| / y}-1-s y^{\alpha}\left(g_{|\varepsilon|}-1\right), \quad 0<\eta_{y}<1 ;
\end{aligned}
$$

here the last term in (4.7) involves two integrands in which the difference quotients for $\lambda=0$ and $\lambda=1$ of $\left(1-s z^{\alpha}+\lambda g^{*}\right)^{p-1}$ and of $y^{\gamma-1}\left(1-s y^{\alpha}+\lambda g^{* *}\right)^{p-1}$ have been expressed according to the law of the mean. Consequently we have

$$
g_{|\varepsilon|} I_{-6}=\left(g_{|\varepsilon|}-1\right) I_{-6}+s I_{10}+(p-1)\left(s I_{-9}-I_{-8}\right) \text {. }
$$

In (4.8) the first term of the second member approaches zero as $\varepsilon \rightarrow 0$, since $g_{|\varepsilon|}-1=O(\varepsilon)$ and $I_{-6}$ is either bounded or $O\left(|\varepsilon|^{\gamma}\right), \gamma>-1$. By (4.1) the parenthesis in $I_{-9}$ is positive except for $z=1, \varepsilon>0$, and indeed lies between its values for $\theta_{z}=0$ and $\theta_{z}=1$, namely lies between $1-s z^{\alpha}$ and $u_{z}$; hence this parenthesis divided by $1-s z^{\alpha}$ is (for sufficiently small $\left.|\varepsilon|\right)$ bounded and bounded from zero; moreover $\left|g^{*} /\left(1-s z^{\alpha}\right)\right|$ is bounded, so $I_{-9}$ converges $(\varepsilon>0, p<1)$. A similar discussion using (4.2) shows that the parenthesis in $I_{-8}$ is positive except for $y=1, \varepsilon>0$, and that $I_{-8}$ converges $(\varepsilon>0, p<1)$.

We now show that $I_{-9} \rightarrow 0$ as $\varepsilon \rightarrow 0$. The law of the mean gives $g^{*} \sim|\varepsilon| g^{\prime}(0)$ $\cdot\left(1-s z^{\alpha+1}\right)$ uniformly in $z$ as $\varepsilon \rightarrow 0$. For $0 \leqq z \leqq 1$ the quotient $\left(1-s z^{\alpha+1}\right) /\left(1-s z^{\alpha}\right)$ is bounded and bounded from zero, so the absolute value of the integrand of $I_{-9}$ is less than $|\varepsilon|$ times a positive constant times $\left(1-s z^{\alpha}\right)^{p-1}$. For $p<1$ the integral from zero to one of the latter function converges, so the assertion concerning $I_{-9}$ follows.

The contribution of $I_{-8}$ to $g_{|\varepsilon|} I_{-6}$ for $p \neq 1$ does not always tend to zero with $\varepsilon$. To assess it, we write $I_{-8}=\int_{|\varepsilon|}{ }^{a}+\int_{a}^{1}, a=|\varepsilon|^{1 / 2}$. For $a \leqq y<1$ we have, with $G_{1}$ as in the beginning of $\S 4.2$, 


$$
\begin{aligned}
\left|g^{* *}\right| & \left.=\mid g_{|\varepsilon|}-1\right)\left(1-s y^{\alpha}\right)+g_{|\varepsilon| / y}-g_{|\varepsilon|} \mid \\
& <\frac{1}{2} G_{1}|\varepsilon|\left(1-s y^{\alpha}\right)+G_{1}(|\varepsilon| / y-|\varepsilon|) \\
& <G_{1}|\varepsilon|\left(1-s y^{\alpha+1}\right) / y \leqq b G_{1} a\left(1-s y^{\alpha}\right), \\
b & =\left(1-s a^{\alpha+1}\right) /\left(1-s a^{\alpha}\right),
\end{aligned}
$$

and for sufficiently small $|\varepsilon|$, by the equation

$$
\begin{aligned}
& 1-s y^{\alpha}+\eta_{y} g^{* *}=\left(1-s y^{\alpha}\right)\left(1+a b G_{1} \sigma_{y}\right), \quad\left|\sigma_{y}\right|<1, \\
& \left|\int_{a}^{1}\right|<b G_{1} a \int_{a}^{1}\left(1+b G_{1} \sigma_{y} a\right)^{p-2}\left(1-s y^{\alpha}\right)^{p-1} y^{\gamma-1} d y .
\end{aligned}
$$

On the other hand, if $|g-1| \leqq G_{0}$ (we can always choose $G_{0}=G_{1} / 2$ ), then for $|\varepsilon| \leqq y \leqq a$ we have, by the definition of $g^{* *},\left|g^{* *}\right|<G_{0}+G_{1}|\varepsilon|$, and if $G_{0}<1$, then for sufficiently small $\varepsilon$

$$
\left|\int_{|\varepsilon|}^{a}\right|<\left(G_{0}+G_{1}|\varepsilon|\right) \int_{|\varepsilon|}^{a}\left[1-s y^{\alpha}+\tau_{y}\left(G_{0}+G_{1}|\varepsilon|\right)\right]^{p-2} y^{\gamma-1} d y, \quad\left|\tau_{y}\right|<1 .
$$

For $\gamma>0$ there follows $I_{-8} \rightarrow 0$ where $\varepsilon \rightarrow 0$. For $\gamma \leqq 0, I_{10}$ becomes infinite as $\varepsilon \rightarrow 0$, and is certain to dominate if $G_{0}<1$ and if in addition

$$
\begin{array}{ll}
G_{0}\left(1+G_{0}\right)^{p-2}<1, & p>2, \\
G_{1}\left(1-G_{0}\right)^{p-2}<1, & p<2 .
\end{array}
$$

It suffices e.g. that $G_{0}<.381$ for $0<p<1, G_{0}<\frac{1}{2}$ for $1 \leqq p<2, G_{0}<.618$ for $2<p \leqq 3, G_{0}<.465$ for $3<p \leqq 4$. In summary we have (in view of $d c / d \varepsilon \sim \alpha|\varepsilon|^{\alpha-1}$ )

THEOREM 11. For a function $f(x)$ fulfilling the assumptions at the beginning of 4.2, the conclusions of Theorem 2 including the Remark and of Theorem 3 hold with the following modifications:

For $\gamma \leqq 0$ we assume in addition $|g-1| \leqq G_{0}<1$, where $G_{0}$ satisfies (4.9).

For $\gamma=1, c>0$, we conclude only $\phi^{\prime}(\varepsilon)=o\left(\varepsilon^{\alpha-2}\right), \phi^{\prime}(0+)=0, \delta^{\prime}(c)=o\left(\varepsilon^{-1}\right)$.

Similarly as in 2.6 there follows

THEOREM 12. For a function $f(x)$ fulfilling the assumptions at the beginning of 4.2 (assuming for $\gamma \leqq 0$ that $|g-1| \leqq G_{0}<1$ with (4.9)) the deviation $\delta(c)$, $|c|<c_{0}$, for sufficiently small $c_{0}$, is a strictly increasing function of $c$ for $\gamma<1$, of $|c|$ for $\gamma>1$.

Thus in the latter case, i.e., for $p<1$ and $\alpha>2 /(1-p)$, zero is a locally best approximating constant for $f(x)$. By defining $f(x)$ only for $0 \leqq x \leqq \varepsilon_{0}$ (where $c_{0}=f\left(\varepsilon_{0}\right)$ ), zero becomes globally best, and the remark concluding 2.6 applies therefore to a much wider class of approximees. 


\section{REFERENCES}

1. P. G. Hoel, Certain problems in the theory of closest approximation, Amer. J. Math. 57 (1935), 891-901.

2. T. S. Motzkin and J. L. Walsh, Least pth power polynomials on a real finite point set, Trans. Amer. Math. Soc. 78 (1955), 67-81.

3. - Polynomials of best approximation on a real finite point set, Trans. Amer. Math. Soc. 91 (1959), 231-245.

4. - A persistent local maximum of the pth power deviation on an interval, $p<1$, (to appear).

5. J. L. Walsh and T. S. Motzkin, Polynomials of best approximation on an interval, Proc. Nat. Acad. Sci. U.S.A. 45 (1959), 1523-1528.

6. - Best approximators within a linear family on an interval, Proc. Nat. Acad. Sci. U.S.A. 46 (1960), 1225-1233.

7. - Polynomials of best approximation on an interval. II, Proc. Nat. Acad. Sci. U.S.A. 48 (1962), 1533-1537.

UNIVERSTTY OF CALIFORNIA,

Los ANgeles, CA Lifornia

HARVARD UNIVERSITY,

CAMBridge, Massachusetts 